\title{
Electroweak pseudo-observables and Z-boson form factors at two-loop accuracy
}

\author{
levgen Dubovyk, ${ }^{a}$ Ayres Freitas, ${ }^{b}$ Janusz Gluza, ${ }^{c, d}$ Tord Riemann $^{c, e}$ and \\ Johann Usovitsch ${ }^{f}$ \\ ${ }^{a}$ II. Institut für Theoretische Physik, Universität Hamburg, \\ 22761 Hamburg, Germany \\ ${ }^{b}$ Pittsburgh Particle Physics, Astrophysics 8 Cosmology Center (PITT PACC), \\ Department of Physics \& Astronomy, University of Pittsburgh, \\ Pittsburgh, PA 15260, U.S.A. \\ ${ }^{c}$ Institute of Physics, University of Silesia, \\ Katowice, Poland \\ ${ }^{d}$ Faculty of Science, University of Hradec Králové, \\ 50003 Hradec Králové, Czech Republic \\ e Deutsches Elektronen-Synchrotron, DESY, \\ 15738 Zeuthen, Germany \\ ${ }^{f}$ School of Mathematics, Trinity College Dublin, University of Dublin, \\ Dublin 2, Ireland \\ E-mail: e.a.dubovyk@gmail.com, afreitas@pitt.edu, gluza@us.edu.pl, \\ tordriemann@gmail.com, jusovitsch@gmail.com
}

ABSTRACT: We present Standard Model predictions for the complete set of phenomenologically relevant electroweak precision pseudo-observables related to the $Z$-boson: the leptonic and bottom-quark effective weak mixing angles $\sin ^{2} \theta_{\text {eff }}^{\ell}, \sin ^{2} \theta_{\text {eff }}^{b}$, the $Z$-boson partial decay widths $\Gamma_{f}$, where $f$ indicates any charged lepton, neutrino and quark flavor (except for the top quark), as well as the total $Z$ decay width $\Gamma_{Z}$, the branching ratios $R_{\ell}, R_{c}, R_{b}$, and the hadronic cross section $\sigma_{\text {had }}^{0}$. The input parameters are the masses $M_{\mathrm{Z}}, M_{\mathrm{H}}$ and $m_{\mathrm{t}}$, and the couplings $\alpha_{\mathrm{s}}, \alpha$. The scheme dependence due to the choice of $M_{\mathrm{W}}$ or its alternative $G_{\mu}$ as a last input parameter is also discussed. Recent substantial technical progress in the calculation of Minkowskian massive higher-order Feynman integrals allows the calculation of the complete electroweak two-loop radiative corrections to all the observables mentioned. QCD contributions are included appropriately. Results are provided in terms of simple and convenient parameterization formulae whose coefficients have been determined from the full numerical multi-loop calculation. The size of the missing electroweak three-loop or QCD higher-order corrections is estimated. We briefly comment on the prospects for their calculation. Finally, direct predictions for the $Z \bar{f} f$ vector and axial-vector form-factors are given, including a discussion of separate order-by-order contributions.

KeYwords: Quark Masses and SM Parameters, Scattering Amplitudes

ArXiv ePrint: 1906.08815 


\section{Contents}

1 Introduction 1

$2 \quad Z$-boson decay width, branching ratios and cross-sections 3

$\begin{array}{lll}3 & \text { Asymmetries and effective weak mixing angles } & 6\end{array}$

4 Vector and axial-vector $Z$-boson form factors $F_{\mathrm{V}}^{f}$ and $F_{\mathrm{A}}^{f} \quad 9$

5 Theoretical error estimates for missing higher order corrections $\quad 12$

6 Conclusions and outlook 13

\section{Introduction}

In 2018 we celebrated 50 years of the Standard Model of elementary particles. The basics of the model were formulated and experimentally validated in the 1960s/70s. The next decade brought an intensive development of the calculation of quantum field theoretical radiative corrections in that model and in its alternatives. An experimental highlight in this context was the $e^{+} e^{-}$-collider LEP, which enabled us to check the Standard Model at an accuracy of better than the per-cent level, which corresponds to effects from more than one electroweak and two QCD loop orders. This proved, for the first time in a systematic way, the Standard Model as a quantum field theory. LEP 1 was running, from Summer 1989 to 1995, at and around the $Z$-boson peak. The expectation for the experimental precision of $M_{\mathrm{Z}}$ and $\Gamma_{\mathrm{Z}}$ was $20 \mathrm{MeV}$ in 1986 [1] and reached finally $2 \mathrm{MeV}$ [2]. This precision tag was extremely important because $M_{Z}$ is one of the Standard Model input parameters to the commonly used on-mass-shell renormalization scheme. Indeed, the experimental accuracy of $M_{\mathrm{Z}}$ triggered much of the precision loop calculations, including the prediction of the top quark and Higgs masses prior to their discoveries from loop corrections to LEP observables in the Standard Model, see refs. [3-5] (as well as refs. [6, 7] for an overview of the current state of the art). Data from the $Z$ peak and the $Z$ resonance curve (the $Z$ line shape) allow to measure a large variety of observables, such as $M_{Z}, \Gamma_{Z}$, cross-sections for different twofermion final states and their ratios and angular asymmetries, together with radiation of (sufficiently soft) photons, gluons, etc. From the real observables, the so-called electroweak pseudo-observables (EWPOs) are extracted by means of a de-convolution of initial-state radiation and subtraction of backgrounds. The fine details of relating EWPOs to real cross-sections at LEP 1 precision are described in detail in ref. [8] and references quoted therein. 
On occasion of the $50^{\text {th }}$ anniversary of the Standard Model, many of the related crucial developments were remembered at the conference "SM@50" [9].

This article is devoted to the state-of-the art calculation of the Standard Model (SM) corrections to the $Z \bar{f} f$-vertex and their inclusion into the predictions for the various EWPOs. We will mostly focus on recent advances in the calculation of the electroweak two-loop terms. QCD contributions, which are known up to four-loop order, have also been taken into account in the results presented here, but we will refer to the literature for further details.

The $Z$ resonance curve can be described theoretically by writing the S-matrix elements as a Laurent series in the center-of-mass energy squared $s$ (also called S-matrix ansatz). This Laurent series ansatz is worked out up to two loops [10-18]. The coefficients of the leading series term contain the $Z$ vertex form factors. Their one-loop corrections were studied in the 1980s; first with massless fermions [19-22], and slightly later with the full mass dependence of the Standard Model [23-26]. After several papers on approximate/partial higher-order corrections, the complete two-loop weak corrections were determined in a series of papers from 2004 to 2018 [27-37]. The correct formulation of the interplay of the $2 \rightarrow 2$ loop corrections with higher order real QED corrections in the S-matrix approach, also called un-folding of the effective $2 \rightarrow 2$ Born terms from the realistic $2 \rightarrow n$ observables, is a topic on its own. It was first studied in refs. [38-41], but its extension beyond two-loop level will require more work $[8,42,43]$. The numerically relevant two-loop and partial higher-order corrections were included in the final analysis of LEP 1 data [44]. ${ }^{1}$

The theoretical advances described here go beyond the Standard Model theory used for physics at LEP $1[45,46]$ but will be needed for the FCC-ee Tera-Z project $[6,7,50-52]$ whose unique experimental precision calls for perturbative predictions at three electroweak loops together with corresponding QCD terms.

In this work, the following pseudo-EWPOs are discussed: the partial widths $\Gamma_{f}$ for $Z$-boson decay into $f \bar{f}$ final states; the total $Z$ width $\Gamma_{Z}$; the branching fractions $\Gamma_{f} / \Gamma_{Z}$; the total hadronic $Z$-pole quark-pair production cross-section $\sigma_{\text {had }}^{0}$; and the effective weak mixing angles $\sin ^{2} \theta_{\text {eff }}^{f}$, defined from the ratio of vector- and axial $Z$-boson couplings. The precise definition of all these pseudo-observables will be given below. Pseudo-observables differ from real observables by removing from the former the effects of initial-state and initial-final QED radiation, as well as non-resonant photon-exchange, box and $t$-channel contributions $[6,44]$.

\footnotetext{
${ }^{1}$ The EWPOs at LEP 1 were determined order by order without a Laurent expansion. This was based on the ZFITTER software [45, 46], for both the Standard Model loop calculation and the unfolding of cross-sections. The relevant higher-order corrections to the input $W$ mass [47] and to the leptonic weak mixing angle [27] are implemented in ZFITTER v.6.42. While ZFITTER v.6.44beta [48] also contains the QCD four-loop corrections of [49], they are of no experimental relevance so far.
} 
The Standard Model predictions for $Z$-pole pseudo-observables can be constructed in terms of the following three theoretical building blocks [29]:

$$
\begin{aligned}
& v_{f}(s) \equiv v_{f}^{\mathrm{Z}}(s)-v_{f}^{\gamma}(s) \frac{\Sigma_{\gamma \mathrm{Z}}(s)}{s+\Sigma_{\gamma \gamma}(s)}, \\
& a_{f}(s) \equiv a_{f}^{\mathrm{Z}}(s)-a_{f}^{\gamma}(s) \frac{\Sigma_{\gamma \mathrm{Z}}(s)}{s+\Sigma_{\gamma \gamma}(s)}, \\
& \Sigma_{\mathrm{Z}}(s) \equiv \Sigma_{\mathrm{ZZ}}(s)-\frac{\left[\Sigma_{\gamma \mathrm{Z}}(s)\right]^{2}}{s+\Sigma_{\gamma \gamma}(s)},
\end{aligned}
$$

where $v_{f}^{Z}$ and $a_{f}^{Z}$ are the one-particle irreducible $Z f \bar{f}$ vector- and axial-vector vertex contributions, respectively, whereas $v_{f}^{\gamma}$ and $a_{f}^{\gamma}$ are their counterparts for the $\gamma f \bar{f}$ vertex. The $\Sigma_{V_{1} V_{2}}$ denotes the one-particle irreducible $V_{1}-V_{2}$ self-energy. At tree level,

$$
\begin{array}{ll}
v_{f(0)}^{\mathrm{Z}}=e \frac{I_{f}^{3}-2 Q_{f} s_{\mathrm{W}}^{2}}{2 s_{\mathrm{W}} c_{\mathrm{W}}}, & v_{f(0)}^{\gamma}=e Q_{f}, \\
a_{f(0)}^{\mathrm{Z}}=e \frac{I_{f}^{3}}{2 s_{\mathrm{W}} c_{\mathrm{W}}}, & a_{f(0)}^{\gamma}=0 .
\end{array}
$$

Here $I_{f}^{3}$ and $Q_{f}$ are the weak isospin and electric charge (in units of the elementary charge $e>0$ ) of the fermion $f$, respectively. $s_{\mathrm{W}}$ and $c_{\mathrm{W}}$ are the sine and cosine of the weak mixing angle, respectively, and the subscript (0) is used to denote tree-level order.

For the theory calculations, these building blocks must be evaluated at the complex $Z$ pole [11-13, 17], $s_{0} \equiv \bar{M}_{\mathrm{Z}}^{2}-i \bar{M}_{\mathrm{Z}} \bar{\Gamma}_{\mathrm{Z}}$, where $\bar{M}_{\mathrm{Z}}$ and $\bar{\Gamma}_{\mathrm{Z}}$ are the on-shell mass and width of the $Z$-boson, respectively. Note that $\bar{M}_{\mathrm{Z}}$ and $\bar{\Gamma}_{\mathrm{Z}}$ differ from the mass $M_{\mathrm{Z}}$ and width $\Gamma_{\mathrm{Z}}$ reported in publications of LEP, Tevatron and LHC experiments by a fixed factor $[38,53]$ :

$$
\begin{gathered}
\bar{M}_{\mathrm{Z}}=M_{\mathrm{Z}} / \sqrt{1+\Gamma_{\mathrm{Z}}^{2} / M_{\mathrm{Z}}^{2}}, \\
\bar{\Gamma}_{\mathrm{Z}}=\Gamma_{\mathrm{Z}} / \sqrt{1+\Gamma_{\mathrm{Z}}^{2} / M_{\mathrm{Z}}^{2}} .
\end{gathered}
$$

Similar expressions hold for $\bar{M}_{\mathrm{W}}$ and $\bar{\Gamma}_{\mathrm{W}}[54,55]$.

\section{$2 \quad Z$-boson decay width, branching ratios and cross-sections}

The width of the $Z$ boson, $\bar{\Gamma}_{Z}$, is related to the imaginary part of the $Z$ self-energy. Using the optical theorem, one can derive the following expression for $\bar{\Gamma}_{\mathrm{Z}}[33,35]$ :

$$
\begin{aligned}
& \bar{\Gamma}_{\mathrm{Z}}=\sum_{f} \bar{\Gamma}_{f}, \quad f=e, \mu, \tau, \nu_{e}, \nu_{\mu}, \nu_{\tau}, u, c, s, d, b, \\
& \bar{\Gamma}_{f}=\frac{N_{c}^{f} \bar{M}_{\mathrm{Z}}}{12 \pi}\left[\mathcal{R}_{\mathrm{V}}^{f} F_{\mathrm{V}}^{f}+\mathcal{R}_{\mathrm{A}}^{f} F_{\mathrm{A}}^{f}\right]_{s=\bar{M}_{\mathrm{Z}}^{2}} .
\end{aligned}
$$

Here $N_{c}^{f}$ is the color factor and $\mathcal{R}_{\mathrm{V}, \mathrm{A}}$ are radiator functions that capture final-state QCD and QED corrections, see section 7 in ref. [56, 57], whereas the remaining electroweak and 
mixed electroweak-QCD corrections are contained in the form factors $F_{\mathrm{V}, \mathrm{A}}^{f}$. Up to two-loop accuracy, the form factors can be written as follows [35]:

$$
\begin{aligned}
& F_{\mathrm{V}}^{f}=\left.\frac{\left|v_{f}\right|^{2}}{1+\operatorname{Re}\left\{\Sigma_{\mathrm{Z}}^{\prime}-\frac{i}{2} \bar{M}_{\mathrm{Z}} \bar{\Gamma}_{\mathrm{Z}} \Sigma_{\mathrm{Z}}^{\prime \prime}\right\}}\right|_{s=\bar{M}_{\mathrm{Z}}^{2}}, \\
& F_{\mathrm{A}}^{f}=\left.\frac{\left|a_{f}\right|^{2}}{1+\operatorname{Re}\left\{\Sigma_{\mathrm{Z}}^{\prime}-\frac{i}{2} \bar{M}_{\mathrm{Z}} \bar{\Gamma}_{\mathrm{Z}} \Sigma_{\mathrm{Z}}^{\prime \prime}\right\}}\right|_{s=\bar{M}_{\mathrm{Z}}^{2}},
\end{aligned}
$$

where $\Sigma_{Z}^{\prime}$ and $\Sigma_{Z}^{\prime \prime}$ are shorthand expressions for $d \Sigma_{\mathrm{Z}} / d s$ and $d^{2} \Sigma_{\mathrm{Z}} / d s^{2}$, respectively.

In addition to the partial widths, certain branching ratios are of phenomenological importance:

$$
R_{\ell}=\bar{\Gamma}_{\text {had }} / \bar{\Gamma}_{\ell}, \quad R_{c}=\bar{\Gamma}_{c} / \bar{\Gamma}_{\text {had }}, \quad R_{b}=\bar{\Gamma}_{b} / \bar{\Gamma}_{\text {had }}
$$

Here $\bar{\Gamma}_{\text {had }}=\sum_{f=u, c, d, s, b} \bar{\Gamma}_{f}$. Further, the cross-section for $e^{+} e^{-} \rightarrow$ hadrons at the $Z$ peak can be expressed in terms of partial widths [35],

$$
\sigma_{\text {had }}=\sigma_{\text {had }}^{0}+\sigma_{\text {had,non-res }}, \quad \sigma_{\text {had }}^{0}=\sum_{f=u, d, c, s, b} \frac{12 \pi}{\bar{M}_{\mathrm{Z}}^{2}} \frac{\bar{\Gamma}_{e} \bar{\Gamma}_{f}}{\bar{\Gamma}_{\mathrm{Z}}^{2}}(1+\delta X) .
$$

Here $\sigma_{\text {had,non-res }}^{0}$ accounts for non-resonant photon-exchange, box and $t$-channel contributions. Furthermore, $\delta X$ occurs from higher-order terms of the Laurent expansion of the full amplitude around the complex pole $s_{0}$. At two-loop order, $\delta X$ can be written as $\delta X_{(2)}=-\left(\operatorname{Im} \Sigma_{\mathrm{Z}(1)}^{\prime}\right)^{2}-2 \bar{\Gamma}_{\mathrm{Z}} \bar{M}_{\mathrm{Z}} \operatorname{Im} \Sigma_{\mathrm{Z}(1)}^{\prime \prime}$, where subscripts $(n)$ indicate the loop order. In the limit $m_{f} \ll M_{\mathrm{W}}(f \neq t)$, it is given by

$$
\delta X_{(2)}=-\left[\frac{\alpha}{s_{\mathrm{W}}^{2} c_{\mathrm{W}}^{2}}\left(\frac{7}{8}-\frac{5}{3} s_{\mathrm{W}}^{2}+\frac{20}{9} s_{\mathrm{W}}^{4}\right)\right]^{2} .
$$

It is important to note that eq. (2.6) assumes that initial-state photon radiation effects have been removed by means of a de-convolution procedure, see e.g. ref. [58].

Results for the partial and total $Z$ widths, branching ratios and $\sigma_{\text {had }}^{0}$ including the full two-loop corrections have first been published in ref. [37]. They can be expressed in simple parameterization formulae, which are adequate for most phenomenological applications. Here, we present slightly more complicated formulae that cover an extended numerical range of input parameters: ${ }^{2}$

$$
\begin{array}{rlrl}
25 \mathrm{GeV} & <M_{\mathrm{H}}<225 \mathrm{GeV}, & 155 \mathrm{GeV}<m_{\mathrm{t}}<192 \mathrm{GeV}, \\
M_{\mathrm{Z}} & =91.1876 \pm 0.0084 \mathrm{GeV}, & & \\
\alpha_{\mathrm{s}} & =0.1184 \pm 0.0050, & \Delta \alpha=0.0590 \pm 0.0005 .
\end{array}
$$

Here $\Delta \alpha$ is the shift in the running electromagnetic coupling $\alpha\left(q^{2}\right)$ from $q^{2}=0$ to $M_{\mathrm{Z}}^{2}$, defined by $\alpha\left(M_{\mathrm{Z}}^{2}\right)=\alpha(0) /(1-\Delta \alpha)$. It can be divided into a leptonic and a hadronic

\footnotetext{
${ }^{2}$ This extended input parameter range is useful for determining indirect constraints on various SM parameters from electroweak precision observables (see e.g. section 10 in ref. [59]), since these indirect bounds often extend over larger intervals than the corresponding direct measurements.
} 
part, $\Delta \alpha=\Delta \alpha_{\text {lept }}+\Delta \alpha_{\text {rad }} . \Delta \alpha_{\text {lept }}$ has been computed to three-loop order [60], whereas $\Delta \alpha_{\text {had }}$ contains non-perturbative hadronic contributions, which are commonly extracted from data [61-63]. We neglect the light fermion masses $m_{f}, f \neq t$, everywhere besides in $\Delta \alpha$ and (at leading power) in the radiator functions $\mathcal{R}_{\mathrm{V} / \mathrm{A}}^{f}$. The $W$ boson mass $M_{\mathrm{W}}$ can be computed from the Fermi constant $G_{\mu}$ (see eqs. (6)-(8) in the arXiv version of ref. [47]) and thus is not listed as an independent input parameter. Both $G_{\mu}$ and $\alpha$, the electromagnetic fine structure constant in the Thomson limit, are known with very small uncertainties, and thus we use their central experimental values [59] without any uncertainty interval.

The fitting formulae for the EWPOs have the form

$$
\begin{aligned}
X= & X_{0}+a_{1} L_{\mathrm{H}}+a_{2} L_{\mathrm{H}}^{2}+a_{3} L_{\mathrm{H}}^{4}+a_{4} \Delta_{\mathrm{H}}+a_{5} \Delta_{\mathrm{t}}+a_{6} \Delta_{\mathrm{t}}^{2}+a_{7} \Delta_{\mathrm{t}} L_{\mathrm{H}}+a_{8} \Delta_{\mathrm{t}} L_{\mathrm{H}}^{2} \\
& +a_{9} \Delta_{\alpha_{\mathrm{s}}}+a_{10} \Delta_{\alpha_{\mathrm{s}}}^{2}+a_{11} \Delta_{\alpha_{\mathrm{s}}} \Delta_{\mathrm{H}}+a_{12} \Delta_{\alpha_{\mathrm{s}}} \Delta_{\mathrm{t}}+a_{13} \Delta_{\alpha}+a_{14} \Delta_{\alpha} \Delta_{\mathrm{H}} \\
& +a_{15} \Delta_{\alpha} \Delta_{t}+a_{16} \Delta_{\mathrm{Z}}, \\
L_{\mathrm{H}}= & \log \frac{M_{\mathrm{H}}}{125.7 \mathrm{GeV}}, \quad \Delta_{\mathrm{H}}=\left(\frac{125.7 \mathrm{GeV}}{M_{\mathrm{H}}}\right)^{4}-1, \quad \Delta_{\mathrm{t}}=\left(\frac{m_{\mathrm{t}}}{173.2 \mathrm{GeV}}\right)^{2}-1, \\
\Delta_{\alpha_{\mathrm{s}}}= & \frac{\alpha_{\mathrm{s}}\left(M_{\mathrm{Z}}\right)}{0.1184}-1, \quad \Delta_{\alpha}=\frac{\Delta \alpha}{0.059}-1, \quad \Delta_{\mathrm{Z}}=\frac{M_{\mathrm{Z}}}{91.1876 \mathrm{GeV}}-1 .
\end{aligned}
$$

The coefficients $X_{0}$ and $a_{1}, \ldots a_{16}$ are obtained from fits to a grid of 8750 data points of the full computation. The latter includes

- Complete one-loop corrections [23], which have been re-computed for this work, and full two-loop [33, 35, 37] electroweak corrections;

- Corrections of order $\mathcal{O}\left(\alpha \alpha_{\mathrm{s}}\right)$ to vector-boson self-energies [64-68], which have been re-evaluated for this work;

- Non-factorizable $\mathcal{O}\left(\alpha \alpha_{\mathrm{s}}\right) Z q \bar{q}$ vertex contributions [69-74], which are not captured in the products $\mathcal{R}_{i}^{f} F_{i}^{f}(i=\mathrm{V}, \mathrm{A})$;

- Higher-loop QCD corrections in the limit of a large top Yukawa coupling $y_{\mathrm{t}}$, of orders $\mathcal{O}\left(\alpha_{\mathrm{t}} \alpha_{\mathrm{s}}^{2}\right)[75,76], \mathcal{O}\left(\alpha_{\mathrm{t}}^{2} \alpha_{\mathrm{s}}\right), \mathcal{O}\left(\alpha_{\mathrm{t}}^{3}\right)[77,78]$, and $\mathcal{O}\left(\alpha_{\mathrm{t}} \alpha_{\mathrm{s}}^{3}\right)[79-81]$, where $\alpha_{\mathrm{t}} \equiv y_{\mathrm{t}}^{2} /(4 \pi)$.

- Final-state QED and QCD radiation effects, which enter through the radiator functions $\mathcal{R}_{\mathrm{V}, \mathrm{A}}$, up to the orders $\mathcal{O}\left(\alpha^{2}\right), \mathcal{O}\left(\alpha \alpha_{\mathrm{s}}\right)$ and $\mathcal{O}\left(\alpha_{\mathrm{s}}^{4}\right)[56,57,82,83]$.

Both the $Z$ vertex corrections as well as the prediction of $M_{\mathrm{W}}$ from $G_{\mu}$ have been computed to this same level of perturbation theory.

Numerical values for the coefficients are given in table 1 . Some of the numbers for $X_{0}$ deviate slightly in the last digit from those in ref. [37]. This is due to the larger grid of input parameters used here, which can exert a pull on the fit parameters. The differences are well within the accuracy quoted in the last column of table 1. 


\begin{tabular}{|l|rrrrrrrrr|}
\hline Observable & \multicolumn{1}{c}{$X_{0}$} & \multicolumn{1}{c}{$a_{1}$} & \multicolumn{1}{c}{$a_{2}$} & \multicolumn{1}{c}{$a_{3}$} & \multicolumn{1}{c}{$a_{4}$} & \multicolumn{1}{c}{$a_{5}$} & \multicolumn{1}{c}{$a_{6}$} & \multicolumn{1}{c}{$a_{7}$} & $a_{8}$ \\
\hline$\Gamma_{e, \mu}[\mathrm{MeV}]$ & 83.983 & -0.1202 & -0.06919 & 0.00383 & 0.0597 & 0.8037 & -0.015 & -0.0195 & 0.0032 \\
$\Gamma_{\tau}[\mathrm{MeV}]$ & 83.793 & -0.1200 & -0.06905 & 0.00382 & 0.0596 & 0.8023 & -0.015 & -0.0195 & 0.0032 \\
$\Gamma_{\nu}[\mathrm{MeV}]$ & 167.176 & -0.1752 & -0.1249 & 0.00595 & 0.1046 & 1.253 & -0.110 & -0.0232 & 0.0064 \\
$\Gamma_{u}[\mathrm{MeV}]$ & 299.994 & -0.6152 & -0.2771 & 0.0174 & 0.2341 & 4.051 & -0.467 & -0.0676 & 0.017 \\
$\Gamma_{c}[\mathrm{MeV}]$ & 299.918 & -0.6152 & -0.2771 & 0.0174 & 0.2340 & 4.051 & -0.467 & -0.0676 & 0.017 \\
$\Gamma_{d, s}[\mathrm{MeV}]$ & 382.829 & -0.6685 & -0.3322 & 0.0193 & 0.2792 & 3.792 & -0.18 & -0.0706 & 0.020 \\
$\Gamma_{b}[\mathrm{MeV}]$ & 375.890 & -0.6017 & -0.3158 & 0.0190 & 0.227 & -2.174 & 0.042 & -0.027 & 0.021 \\
$\Gamma_{\mathrm{Z}}[\mathrm{MeV}]$ & 2494.75 & -4.055 & -2.117 & 0.122 & 1.746 & 19.68 & -1.63 & -0.432 & 0.12 \\
\hline$R_{\ell}\left[10^{-3}\right]$ & 20751.6 & -8.112 & -1.174 & 0.155 & 0.16 & -37.59 & -10.9 & 1.27 & 0.29 \\
$R_{c}\left[10^{-5}\right]$ & 17222.2 & -4.049 & -0.749 & 0.0832 & 1.08 & 98.956 & -15.1 & -0.761 & 0.080 \\
$R_{b}\left[10^{-5}\right]$ & 21585.0 & 4.904 & 0.9149 & -0.0535 & -2.676 & -292.21 & 20.0 & 1.97 & -0.11 \\
\hline$\sigma_{\text {had }}^{0}[\mathrm{pb}]$ & 41489.6 & 0.408 & -0.320 & 0.0424 & 1.32 & 60.17 & 16.3 & -2.31 & -0.19 \\
\hline
\end{tabular}

\begin{tabular}{|c|c|c|c|c|c|c|c|c|c|}
\hline Observable & $a_{9}$ & $a_{10}$ & $a_{11}$ & $a_{12}$ & $a_{13}$ & $a_{14}$ & $a_{15}$ & $a_{16}$ & max. dev. \\
\hline$\Gamma_{e, \mu}[\mathrm{MeV}]$ & -0.0956 & -0.0078 & -0.0095 & 0.25 & -1.08 & 0.056 & -0.37 & 286 & $<0.0015$ \\
\hline$\Gamma_{\tau}[\mathrm{MeV}]$ & -0.0954 & -0.0078 & -0.0094 & 0.25 & -1.08 & 0.056 & -0.37 & 285 & $<0.0015$ \\
\hline$\Gamma_{\nu}[\mathrm{MeV}]$ & -0.187 & -0.014 & -0.014 & 0.37 & -0.085 & 0.054 & -0.30 & 503 & $<0.002$ \\
\hline$\Gamma_{u}[\mathrm{MeV}]$ & 14.26 & 1.6 & -0.046 & 1.82 & -11.1 & 0.16 & -1.0 & 1253 & $<0.006$ \\
\hline$\Gamma_{c}[\mathrm{MeV}]$ & 14.26 & 1.6 & -0.046 & 1.82 & -11.1 & 0.16 & -1.0 & 1252 & $<0.006$ \\
\hline$\Gamma_{d, s}[\mathrm{MeV}]$ & 10.20 & -2.4 & -0.052 & 0.71 & -10.1 & 0.16 & -0.92 & 1469 & $<0.007$ \\
\hline$\Gamma_{b}[\mathrm{MeV}]$ & 10.53 & -2.4 & -0.056 & 1.2 & -10.1 & 0.15 & -0.95 & 1458 & $<0.007$ \\
\hline$\Gamma_{\mathrm{Z}}[\mathrm{MeV}]$ & 58.61 & -4.0 & -0.32 & 8.1 & -56.1 & 1.1 & -6.8 & 9267 & $<0.04$ \\
\hline$R_{\ell}\left[10^{-3}\right]$ & 732.30 & -44 & -0.61 & 5.7 & -358 & -4.7 & 37 & 11649 & $<0.12$ \\
\hline$R_{c}\left[10^{-5}\right]$ & 230.9 & 125 & 0.045 & 36.9 & -120 & 1.2 & -6.2 & 3667 & $<0.1$ \\
\hline$R_{b}\left[10^{-5}\right]$ & -131.9 & -84 & -0.27 & 4.4 & 71.9 & -0.77 & -4.4 & -1790 & $<0.12$ \\
\hline$\sigma_{\text {had }}^{0}[\mathrm{pb}]$ & -579.58 & 38 & 0.010 & 7.5 & 85.2 & 9.1 & -68 & -85957 & $<0.15$ \\
\hline
\end{tabular}

Table 1. Coefficients for the parameterization formula (2.9) for various observables. Within the ranges $25 \mathrm{GeV}<M_{\mathrm{H}}<225 \mathrm{GeV}, 155 \mathrm{GeV}<m_{\mathrm{t}}<195 \mathrm{GeV}, \alpha_{\mathrm{s}}=0.1184 \pm 0.0050, \Delta \alpha=$ $0.0590 \pm 0.0005$ and $M_{\mathrm{Z}}=91.1876 \pm 0.0084 \mathrm{GeV}$, the formulae approximate the full result with maximal deviations given in the last column.

\section{$3 \quad$ Asymmetries and effective weak mixing angles}

The effective weak mixing angle for the $Z f \bar{f}$ vertex is defined, from the theory side, as

$$
\sin ^{2} \theta_{\mathrm{eff}}^{f} \equiv \frac{1}{4\left|Q_{f}\right|}\left(1+\operatorname{Re}\left\{\frac{v_{f}\left(\bar{M}_{\mathrm{Z}}^{2}\right)}{a_{f}\left(\bar{M}_{\mathrm{Z}}^{2}\right)}\right\}\right) .
$$

Here $\bar{M}_{\mathrm{W}}^{2}$ and $\bar{M}_{\mathrm{Z}}^{2}$ are the real parts of the complex pole of the $W$ and $Z$ propagators, respectively. They are related to the masses commonly reported by experiments at LEP, 


\begin{tabular}{|c|c|c|}
\hline$M_{\mathrm{H}}[\mathrm{GeV}]$ & Result of ref. [28] & Our result \\
\hline 100 & $-0.733989 \times 10^{-4}$ & $-0.733955 \times 10^{-4}$ \\
200 & $-0.469470 \times 10^{-4}$ & $-0.471273 \times 10^{-4}$ \\
\hline
\end{tabular}

Table 2. Comparison of numerical results for $\Delta \kappa_{\ell}^{\left(\alpha^{2} \text {,bos }\right)}$ from ref. [28] with our calculation of $Z$ vertex corrections from ref. [37]. We use $\bar{M}_{\mathrm{Z}}=91.1876 \mathrm{GeV}$ and $\bar{M}_{\mathrm{W}}=80.385 \mathrm{GeV}$.

Tevatron, LHC according to eq (1.6). Moreover, $Q_{f}$ denotes the electric charge of the fermion $f$.

The effective weak mixing angles can be extracted from a range of asymmetries [8], defined from effective Born two-particle cross-sections, including the left-right asymmetry

$$
A_{\mathrm{LR}}=\frac{\sigma_{e_{\mathrm{L}}}-\sigma_{e_{\mathrm{R}}}}{\sigma_{e_{\mathrm{L}}}+\sigma_{e_{\mathrm{R}}}}=A_{e}+A_{\mathrm{LR}}^{\mathrm{non}-\mathrm{res}}
$$

and the forward-backward asymmetry

$$
A_{\mathrm{FB}}^{f}=\frac{\sigma_{\cos \theta>0}-\sigma_{\cos \theta<0}}{\sigma_{\cos \theta>0}+\sigma_{\cos \theta<0}}=\frac{3}{4} A_{e} A_{f}+A_{\mathrm{FB}}^{f, \text { non-res }},
$$

where

$$
A_{f} \equiv \frac{2 \operatorname{Re}\left\{v_{f} / a_{f}\right\}}{1+\left(\operatorname{Re}\left\{v_{f} / a_{f}\right\}\right)^{2}}=\frac{1-4\left|Q_{f}\right| \sin ^{2} \theta_{\mathrm{eff}}^{f}}{1-4\left|Q_{f}\right| \sin ^{2} \theta_{\mathrm{eff}}^{f}+8\left(\left|Q_{f}\right| \sin ^{2} \theta_{\mathrm{eff}}^{f}\right)^{2}} .
$$

Here $\sigma_{e_{\mathrm{L}}}$ and $\sigma_{e_{\mathrm{R}}}$ are the cross-sections for $e^{+} e^{-} \rightarrow f \bar{f}$ for left- and right-handed polarized electron beams, respectively, whereas $\sigma_{\cos \theta>0}$ and $\sigma_{\cos \theta<0}$ denote the cross-section for $f$ restricted to the forward and backward hemisphere, respectively. Furthermore, $A_{X}^{\text {non-res }}$ accounts for the non-resonant photon-exchange, box and $t$-channel contributions.

The most precisely measured effective weak mixing angles are the leptonic effective weak mixing angle $\sin ^{2} \theta_{\text {eff }}^{\ell}$ (extracted from $A_{\mathrm{LR}}$ ) and the bottom-quark one, $\sin ^{2} \theta_{\text {eff }}^{b}$ (extracted from $\left.A_{\mathrm{FB}}^{b}\right)[58]$.

Standard Model predictions for $\sin ^{2} \theta_{\text {eff }}^{\ell}$ including the full two-loop corrections have been presented originally in refs. $[28,29,31]$. We reproduced by an independent calculation the contribution of the bosonic electroweak two-loop corrections using the methods of ref. [37]. The corrections can be expressed in terms of a weak form factor $\Delta \kappa_{\ell}^{\left(\alpha^{2}, \text { bos }\right)}$, where

$$
\Delta \kappa_{f}=\left(1-\frac{\bar{M}_{\mathrm{W}}}{\bar{M}_{\mathrm{Z}}}\right)^{-1} \sin ^{2} \theta_{\mathrm{eff}}^{f}-1
$$

The comparison with ref. [28] is shown in table 2, which demonstrates that the two calculations agree to an accuracy of $\mathcal{O}\left(10^{-7}\right)$, which implies an accuracy of better than $10^{-7}$ for $\sin ^{2} \theta_{\text {eff }}^{\ell}$. The full two-loop corrections for $\sin ^{2} \theta_{\text {eff }}^{b}$ have been presented first in ref. [36].

In the following, we present simple parameterization formulae for $\sin ^{2} \theta_{\mathrm{eff}}^{\ell}$ and $\sin ^{2} \theta_{\mathrm{eff}}^{b}$, which cover the extended range of input parameters of eq. (2.8). The parameterization 
formula

$$
\begin{aligned}
\sin ^{2} \theta_{\mathrm{eff}}^{f}= & s_{0}+d_{1} L_{H}+d_{2} L_{H}^{2}+d_{3} L_{H}^{4}+d_{4} \Delta_{\alpha}+d_{5} \Delta_{\mathrm{t}}+d_{6} \Delta_{\mathrm{t}}^{2}+d_{7} \Delta_{\mathrm{t}} L_{H} \\
& +d_{8} \Delta_{\alpha_{\mathrm{s}}}+d_{9} \Delta_{\alpha_{\mathrm{s}}} \Delta_{\mathrm{t}}+d_{10} \Delta_{\mathrm{Z}}
\end{aligned}
$$

with

$$
\begin{aligned}
L_{\mathrm{H}} & =\log \frac{M_{\mathrm{H}}}{125.7 \mathrm{GeV}}, & \Delta_{\mathrm{t}} & =\left(\frac{m_{\mathrm{t}}}{173.2 \mathrm{GeV}}\right)^{2}-1, \\
\Delta_{\alpha_{\mathrm{s}}} & =\frac{\alpha_{\mathrm{s}}\left(M_{\mathrm{Z}}\right)}{0.1184}-1, & \Delta_{\alpha} & =\frac{\Delta \alpha}{0.059}-1,
\end{aligned}
$$

provides a good description of the full result in the parameter region (2.8). Values for the coefficients are obtained by fitting (3.6) to a grid of 8750 data points.

Table 3 shows the result of a fit to a calculation that includes all known corrections:

- Complete one- and two-loop electroweak corrections,

(see refs. [21, 23, 27, 28, 30-32, 36] for the original references);

- Corrections of order $\mathcal{O}\left(\alpha \alpha_{\mathrm{s}}\right)$ to vector-boson self-energies [64-68], which we have re-evaluated for this work;

- Non-factorizable $\mathcal{O}\left(\alpha \alpha_{\mathrm{s}}\right) Z b \bar{b}$ vertex contributions [69-74], which do not cancel in the ratio $v_{b} / a_{b}$;

- Higher-loop corrections in the limit of a large top Yukawa coupling $y_{\mathrm{t}}$, of orders $\mathcal{O}\left(\alpha_{\mathrm{t}} \alpha_{\mathrm{s}}^{2}\right)[75,76], \mathcal{O}\left(\alpha_{\mathrm{t}}^{2} \alpha_{\mathrm{s}}\right), \mathcal{O}\left(\alpha_{\mathrm{t}}^{3}\right)[77,78]$, and $\mathcal{O}\left(\alpha_{\mathrm{t}} \alpha_{\mathrm{s}}^{3}\right)[79-81]$ where $\alpha_{\mathrm{t}} \equiv y_{\mathrm{t}}^{2} /(4 \pi)$.

As indicated by the last column in the table, the largest deviation of the fit formulae from the full result is $\mathcal{O}\left(\right.$ few $\left.\times 10^{-6}\right)$, while for most of the parameter region in (2.8) the agreement is better than $10^{-6}$. The careful reader may realize that the parameterization for $\sin ^{2} \theta_{\text {eff }}^{b}$ in table 3 deviates slightly from eqs. $(20,22)$ in [36]. The difference is due to the larger grid of data points used here. A fit formula is, obviously, not able to reproduce the data points in a grid perfectly. The fitting aims to find the best average agreement between the data points (which are generated with our full numerical calculation) and the fit formula. A larger grid therefore can lead to some shifts of the coefficients. As a consequence, the formula in [36] will probably be more accurate for input values within the ranges in table 1 there. On the other hand, while the formula here may be a little less accurate within these ranges, it covers a much larger range of input values.

It should also be noted that the fit formula for $\sin ^{2} \theta_{\mathrm{eff}}^{\ell}$ in ref. [28] does not include the $\mathcal{O}\left(\alpha_{\mathrm{t}} \alpha_{\mathrm{s}}^{3}\right)$ corrections from refs. [79-81], but they are included in the formula presented here.

In table 4 it is shown that the technical accuracy of our fit formulae is adequate for the expected experimental precision of several future $e^{+} e^{-}$colliders, although it will get modified by anticipated future three-loop electroweak corrections. 


\begin{tabular}{|l|ccccccc|}
\hline Observable & $s_{0}$ & $d_{1}$ & $d_{2}$ & $d_{3}$ & $d_{4}$ & $d_{5}$ \\
\hline $\sin ^{2} \theta_{\text {eff }}^{\ell} \times 10^{4}$ & 2314.64 & 4.616 & 0.539 & -0.0737 & 206 & -25.71 \\
$\sin ^{2} \theta_{\text {eff }}^{b} \times 10^{4}$ & 2327.04 & 4.638 & 0.558 & -0.0700 & 207 & -9.554 \\
\hline Observable & $d_{6}$ & $d_{7}$ & $d_{8}$ & $d_{9}$ & $d_{10}$ & max. dev. \\
\hline $\sin ^{2} \theta_{\text {eff }}^{\ell} \times 10^{4}$ & 4.00 & 0.288 & 3.88 & -6.49 & -6560 & $<0.056$ \\
$\sin ^{2} \theta_{\text {eff }}^{b} \times 10^{4}$ & 3.83 & 0.179 & 2.41 & -8.24 & -6630 & $<0.025$ \\
\hline
\end{tabular}

Table 3. Coefficients for the parameterization formula (3.6) for the leptonic and bottom-quark effective weak mixing angles. Within the ranges given in eq. (2.8), the formula deviates from the full result up to the maximal amount given in the last column.

\begin{tabular}{|l|ccccc|}
\hline Observable & max. dev. & EXP now & FCC-ee & CEPC & GigaZ \\
\hline$\Gamma_{Z}[\mathrm{MeV}]$ & 0.04 & 2.3 & 0.1 & 0.5 & 0.8 \\
$\sin ^{2} \theta_{\text {eff }}^{\ell} \times 10^{4}$ & 0.056 & 1.6 & 0.06 & 0.23 & 0.1 \\
$\sin ^{2} \theta_{\text {eff }}^{b} \times 10^{4}$ & 0.025 & 160 & 9 & 9 & 15 \\
\hline
\end{tabular}

Table 4. Goodness of fit for some chosen EWPOs, compared with the envisaged precision measurements for $\Gamma_{Z}$ and $\sin ^{2} \theta_{\text {eff }}^{\ell}$ (statistical errors), and $\sin ^{2} \theta_{\text {eff }}^{b}$ (systematic errors) at the collider projects FCC-ee Tera-Z [84], CEPC [85] and ILC/GigaZ [86]. The values of maximal deviations are taken from tables 1 and 3. The entry "EXP now" gives the present experimental precision, as known since LEP 1 [44].

\section{Vector and axial-vector $Z$-boson form factors $F_{\mathrm{V}}^{f}$ and $F_{\mathrm{A}}^{f}$}

The pseudo-observables discussed in the previous sections aim to be closely related to actual observables, such as cross-sections, branching ratios, or asymmetries. On the other hand, for some purposes it is also useful to have numerical results for the underlying vertex corrections themselves [34], for example: (i) Inclusion of selected corrections from Beyond Standard Model (BSM) physics, (ii) Estimations of magnitudes of selected single terms, (iii) Partial cross-checks with other calculations. For such purposes, the form factors $F_{\mathrm{V}}^{f}$ and $F_{\mathrm{A}}^{f}$ introduced in eq. (2.2) are needed explicitly.

Tables 5 and 6 show the numerical contributions of different orders of perturbation theory to $F_{\mathrm{V}}^{f}$ and $F_{\mathrm{A}}^{f}$. Here the form factors are always understood to include the appropriate (on-shell) counterterms to render them UV-finite. In table 5 these are computed using the following input values:

$$
\begin{aligned}
& M_{\mathrm{Z}}=91.1876 \mathrm{GeV}, \quad \Gamma_{\mathrm{Z}}=2.4952 \mathrm{GeV}, \quad \Rightarrow \quad \bar{M}_{\mathrm{Z}}=91.1535 \mathrm{GeV} \\
& M_{\mathrm{W}}=80.385 \mathrm{GeV}, \quad \Gamma_{\mathrm{W}}=2.085 \mathrm{GeV}, \quad \Rightarrow \quad \bar{M}_{\mathrm{W}}=80.358 \mathrm{GeV} \\
& M_{\mathrm{H}}=125.1 \mathrm{GeV}, \quad m_{\mathrm{t}}=173.2 \mathrm{GeV}, \\
& m_{\mathrm{b}}^{\overline{\mathrm{MS}}}=4.2 \mathrm{GeV}, \quad \Delta \alpha=0.059, \quad \alpha_{\mathrm{s}}=0.1184
\end{aligned}
$$

For table 6, on the other hand, the Fermi constant $G_{\mu}$ is used as an input instead of (4.1b), 


\begin{tabular}{|l|rrrcccc|}
\hline Form fact. & Born & $\mathcal{O}(\alpha)$ & $\mathcal{O}\left(\alpha \alpha_{\mathrm{s}}\right)$ & $\begin{array}{c}\mathcal{O}\left(\alpha_{\mathrm{t}} \alpha_{\mathrm{s}}^{2}, \alpha_{\mathrm{t}} \alpha_{\mathrm{s}}^{3},\right. \\
\left.\alpha_{\mathrm{t}}^{2} \alpha_{\mathrm{s}}, \alpha_{\mathrm{t}}^{3}\right)\end{array}$ & $\mathcal{O}\left(N_{f}^{2} \alpha^{2}\right)$ & $\mathcal{O}\left(N_{f} \alpha^{2}\right)$ & $\mathcal{O}\left(\alpha_{\text {bos }}^{2}\right)$ \\
\hline$F_{V}^{\ell}\left[10^{-5}\right]$ & 39.07 & -24.86 & 2.41 & 0.35 & 1.47 & 2.37 & 0.27 \\
$F_{A}^{\ell}\left[10^{-5}\right]$ & 3309.44 & 118.59 & 9.46 & 1.22 & 8.60 & 2.60 & 0.45 \\
$F_{V, A}^{\nu}\left[10^{-5}\right]$ & 3309.44 & 127.56 & 9.46 & 1.22 & 8.60 & 3.83 & 0.39 \\
$F_{V}^{u, c}\left[10^{-5}\right]$ & 544.88 & -44.80 & 7.29 & 1.02 & -1.67 & 3.25 & 0.33 \\
$F_{A}^{u, c}\left[10^{-5}\right]$ & 3309.44 & 120.79 & 9.46 & 1.22 & 8.60 & 3.27 & 0.44 \\
$F_{V}^{d, s}\left[10^{-5}\right]$ & 1635.01 & 5.84 & 9.64 & 1.32 & 0.71 & 3.45 & 0.37 \\
$F_{A}^{d, s}\left[10^{-5}\right]$ & 3309.44 & 123.78 & 9.46 & 1.22 & 8.60 & 3.11 & 0.42 \\
$F_{V}^{b}\left[10^{-5}\right]$ & 1635.01 & -26.16 & 9.64 & 1.32 & 0.71 & 1.77 & 1.05 \\
$F_{A}^{b}\left[10^{-5}\right]$ & 3309.44 & 78.26 & 9.46 & 1.22 & 8.60 & 0.13 & 1.18 \\
\hline
\end{tabular}

Table 5. Contributions of different perturbative orders to the $Z$ vertex form factors. A fixed value of $M_{\mathrm{W}}$ has been used as input, instead of $G_{\mu} . N_{f}^{n}$ refers to corrections with $n$ closed fermions loops, whereas $\alpha_{\text {bos }}^{2}$ denotes corrections without closed fermions loops. Furthermore, $\alpha_{\mathrm{t}}=y_{\mathrm{t}} /(4 \pi)$ where $y_{\mathrm{t}}$ is the top Yukawa coupling.

\begin{tabular}{|l|rrrccc|}
\hline Form fact. & Born & $\mathcal{O}(\alpha)$ & $\mathcal{O}\left(\alpha \alpha_{\mathrm{s}}\right)$ & $\begin{array}{c}\mathcal{O}\left(\alpha_{\mathrm{t}} \alpha_{\mathrm{s}}^{2}, \alpha_{\mathrm{t}} \alpha_{\mathrm{s}}^{3},\right. \\
\left.\alpha_{\mathrm{t}}^{2} \alpha_{\mathrm{s}}, \alpha_{\mathrm{t}}^{3}\right)\end{array}$ & $\mathcal{O}\left(N_{f}^{2} \alpha^{2}, N_{f} \alpha^{2}\right)$ & $\mathcal{O}\left(\alpha_{\text {bos }}^{2}\right)$ \\
\hline$F_{V}^{\ell}\left[10^{-5}\right]$ & 77.63 & -59.86 & 0.31 & -0.09 & 1.88 & 0.24 \\
$F_{A}^{\ell}\left[10^{-5}\right]$ & 3426.43 & 19.32 & -1.12 & -0.92 & 1.62 & 0.28 \\
$F_{V, A}^{\nu}\left[10^{-5}\right]$ & 3426.43 & 28.36 & -1.16 & -0.93 & 2.81 & 0.21 \\
$F_{V}^{u, c}\left[10^{-5}\right]$ & 644.45 & -129.87 & -1.36 & -0.73 & -6.26 & 0.19 \\
$F_{A}^{u, c}\left[10^{-5}\right]$ & 3426.43 & 21.54 & -1.13 & -0.93 & 2.28 & 0.27 \\
$F_{V}^{d, s}\left[10^{-5}\right]$ & 1760.71 & -100.64 & -1.15 & -1.01 & -6.24 & 0.19 \\
$F_{A}^{d, s}\left[10^{-5}\right]$ & 3426.43 & 24.56 & -2.21 & -0.93 & 2.10 & 0.25 \\
$F_{V}^{b}\left[10^{-5}\right]$ & 1760.71 & -133.08 & -1.58 & -0.95 & -7.68 & 0.86 \\
$F_{A}^{b}\left[10^{-5}\right]$ & 3426.43 & -21.45 & -0.85 & -0.87 & -0.62 & 1.01 \\
\hline
\end{tabular}

Table 6. Same as table 5 , but with $M_{\mathrm{W}}$ calculated from $G_{\mu}$.

and $\bar{M}_{\mathrm{W}}$ is computed from

$$
\bar{M}_{\mathrm{W}}^{2}\left(1-\frac{\bar{M}_{\mathrm{W}}^{2}}{\bar{M}_{\mathrm{Z}}^{2}}\right)=\frac{\pi \alpha}{\sqrt{2} G_{\mu}}(1+\Delta r),
$$

where $\Delta r$ has been evaluated to the same orders as given in each column of the table. More details about the calculation of $\Delta r$ can be found in ref. [47]. As before, the dependence of the Standard Model prediction on various input parameters can be expressed in terms of the simple parameterization formula eq. (2.9).

Table 7 shows the numerical values for the coefficients obtained by fitting this formula to the currently most precise computation, including the same corrections as in section 2 , 


\begin{tabular}{|l|rrrrrrrrr|}
\hline Form fact. & \multicolumn{1}{|c}{$X_{0}$} & \multicolumn{1}{c}{$a_{1}$} & \multicolumn{1}{c}{$a_{2}$} & \multicolumn{1}{c}{$a_{3}$} & \multicolumn{1}{c}{$a_{4}$} & \multicolumn{1}{c}{$a_{5}$} & \multicolumn{1}{c}{$a_{6}$} & \multicolumn{1}{c|}{$a_{7}$} & \multicolumn{1}{c|}{$a_{8}$} \\
\hline$F_{V}^{\ell}\left[10^{-5}\right]$ & 20.11 & -1.317 & -0.2615 & 0.0333 & 0.276 & 7.474 & 1.55 & -0.326 & 0.0012 \\
$F_{A}^{\ell}\left[10^{-5}\right]$ & 3446.88 & -3.645 & -2.595 & 0.125 & 2.188 & 25.70 & -2.17 & -0.480 & 0.13 \\
$F_{V, A}^{\nu}\left[10^{-5}\right]$ & 3457.02 & -3.623 & -2.584 & 0.123 & 2.163 & 25.92 & -2.27 & -0.481 & 0.13 \\
$F_{V}^{u, c}\left[10^{-5}\right]$ & 506.214 & -4.485 & -1.063 & 0.105 & 0.912 & 23.21 & -1.54 & -0.410 & 0.085 \\
$F_{A}^{u, c}\left[10^{-5}\right]$ & 3448.76 & -3.635 & -2.591 & 0.124 & 2.181 & 25.83 & -2.24 & -0.480 & 0.13 \\
$F_{V}^{d, s}\left[10^{-5}\right]$ & 1650.98 & -5.247 & -1.836 & 0.134 & 1.541 & 29.22 & -2.78 & -0.458 & 0.13 \\
$F_{A}^{d, s}\left[10^{-5}\right]$ & 3451.41 & -3.617 & -2.584 & 0.123 & 2.170 & 25.67 & -2.21 & -0.477 & 0.13 \\
$F_{V}^{b}\left[10^{-5}\right]$ & 1622.01 & -4.891 & -1.758 & 0.134 & 1.27 & -2.87 & -1.5 & -0.21 & 0.13 \\
$F_{A}^{b}\left[10^{-5}\right]$ & 3409.36 & -3.114 & -2.461 & 0.120 & 1.757 & -21.03 & -0.60 & -0.15 & 0.14 \\
\hline
\end{tabular}

\begin{tabular}{|l|cccccccc|c|}
\hline Form fact. & $a_{9}$ & \multicolumn{1}{c}{$a_{10}$} & $a_{11}$ & $a_{12}$ & \multicolumn{1}{c}{$a_{13}$} & $a_{14}$ & $a_{15}$ & $a_{16}$ & max. dev. \\
\hline$F_{V}^{\ell}\left[10^{-5}\right]$ & -0.13 & -0.037 & -0.11 & 2.78 & -43.17 & 1.2 & -9.0 & 1397 & $<0.02$ \\
$F_{A}^{\ell}\left[10^{-5}\right]$ & -3.81 & -0.29 & -0.28 & 7.6 & -2.0 & 1.1 & -6.3 & 6921 & $<0.04$ \\
$F_{V, A}^{\nu}\left[10^{-5}\right]$ & -3.87 & -0.29 & -0.28 & 7.6 & -1.7 & 1.1 & -6.3 & 6949 & $<0.04$ \\
$F_{V}^{u, c}\left[10^{-5}\right]$ & -3.89 & -0.16 & -0.22 & 6.5 & -145.7 & 0.98 & -6.9 & 5651 & $<0.03$ \\
$F_{A}^{u, c}\left[10^{-5}\right]$ & -4.80 & -0.27 & -0.27 & 7.5 & -1.9 & 1.1 & -6.3 & 6928 & $<0.04$ \\
$F_{V}^{d, s}\left[10^{-5}\right]$ & -5.53 & -0.24 & -0.28 & 8.1 & -132. & 0.95 & -6.0 & 7498 & $<0.04$ \\
$F_{A}^{d, s}\left[10^{-5}\right]$ & -4.98 & -0.27 & -0.27 & 7.5 & -1.7 & 1.1 & -6.2 & 6933 & $<0.04$ \\
$F_{V}^{b}\left[10^{-5}\right]$ & -1.3 & -0.28 & -0.32 & 12.5 & -131. & 0.95 & -5.7 & 7457 & $<0.04$ \\
$F_{A}^{b}\left[10^{-5}\right]$ & 0.93 & -0.31 & -0.31 & 13.6 & -2.5 & 1.1 & -7.2 & 6927 & $<0.04$ \\
\hline
\end{tabular}

Table 7. Coefficients for the parameterization formula (2.9) for various form factors $(X)$. Within the ranges $25 \mathrm{GeV}<M_{\mathrm{H}}<225 \mathrm{GeV}, 155 \mathrm{GeV}<m_{\mathrm{t}}<195 \mathrm{GeV}, \alpha_{\mathrm{s}}=0.1184 \pm 0.0050, \Delta \alpha=$ $0.0590 \pm 0.0005$ and $M_{\mathrm{Z}}=91.1876 \pm 0.0084 \mathrm{GeV}$, the formula approximates the full result with average and maximal deviations given in the last two columns.

except for the final-state QED and QCD radiation effects, i.e.

$$
\begin{aligned}
& F_{\mathrm{V}}^{f}=\left|v_{f(0)}\right|^{2}+F_{\mathrm{V}(\alpha)}^{f}+F_{\mathrm{V}\left(\alpha \alpha_{\mathrm{s}}\right)}^{f}+F_{\mathrm{V}\left(\alpha^{2}\right)}^{f}+F_{\mathrm{V}\left(\alpha_{\mathrm{t}} \alpha_{\mathrm{s}}^{2}\right)}^{f}+F_{\mathrm{V}\left(\alpha_{\mathrm{t}}^{2} \alpha_{\mathrm{s}}\right)}^{f}+F_{\mathrm{V}\left(\alpha_{\mathrm{t}}^{3}\right)}^{f}+F_{\mathrm{V}\left(\alpha_{\mathrm{t}} \alpha_{\mathrm{s}}^{3}\right)}^{f}, \\
& F_{\mathrm{A}}^{f}=\left|a_{f(0)}\right|^{2}+F_{\mathrm{A}(\alpha)}^{f}+F_{\mathrm{A}\left(\alpha \alpha_{\mathrm{s}}\right)}^{f}+F_{\mathrm{A}\left(\alpha^{2}\right)}^{f}+F_{\mathrm{A}\left(\alpha_{\mathrm{t}} \alpha_{\mathrm{s}}^{2}\right)}^{f}+F_{\mathrm{A}\left(\alpha_{\mathrm{t}}^{2} \alpha_{\mathrm{s}}\right)}^{f}+F_{\mathrm{A}\left(\alpha_{\mathrm{t}}^{3}\right)}^{f}+F_{\mathrm{A}\left(\alpha_{\mathrm{t}} \alpha_{\mathrm{s}}^{3}\right)}^{f} .
\end{aligned}
$$

Note that $G_{\mu}$ (rather than $M_{\mathrm{W}}$ ) has been used as one input in table 7 .

The form factor results presented here can be easily augmented to include the effects of some new physics model:

$$
\begin{aligned}
& F_{\mathrm{V}, \mathrm{SM}+\mathrm{NP}}^{f} \approx F_{\mathrm{V}, \mathrm{SM}}^{f}+2 \operatorname{Re}\left\{v_{f(0)} v_{f, \mathrm{NP}}\right\}, \\
& F_{\mathrm{A}, \mathrm{SM}+\mathrm{NP}}^{f} \approx F_{\mathrm{A}, \mathrm{SM}}^{f}+2 \operatorname{Re}\left\{a_{f(0)} a_{f, \mathrm{NP}}\right\} .
\end{aligned}
$$

Here "SM" denotes the SM contributions discussed in the present paper, while "NP" stands for the new physics correction on top of the SM. Since the existing experimental 
constraints imply that any possible new physics effect is small, it is sufficient to use the tree-level couplings $v_{f(0)}$ and $a_{f(0)}$ in the interference terms and neglect the $\left|v_{f, \mathrm{NP}}\right|^{2}$ and $\left|a_{f, \mathrm{NP}}\right|^{2}$ terms.

\section{Theoretical error estimates for missing higher order corrections}

The main theory uncertainty of the results presented in this paper stems from unknown three- and four-loop corrections. The leading missing orders are $\mathcal{O}\left(\alpha^{3}\right), \mathcal{O}\left(\alpha^{2} \alpha_{\mathrm{s}}\right), \mathcal{O}\left(\alpha \alpha_{\mathrm{s}}^{2}\right)$, and $\mathcal{O}\left(\alpha \alpha_{\mathrm{s}}^{3}\right)$. Partial results for these contributions, in the limit of a large top Yukawa coupling $y_{\mathrm{t}}$, have already been computed [75-81]. Therefore, when evaluating the impact of theory uncertainties, it is always implied that we refer to these contributions beyond the leading- $y_{\mathrm{t}}$ limit.

There are a number of different methods for assessing theory uncertainties from unknown higher orders, none of which is fully reliable. Rather, they should be taken as an order-of-magnitude estimate of the size of these terms. A convenient and widely applicable method is based on the assumption that the first few orders of the perturbation series approximately follow a geometric series $[35,37,87]$. In this way one obtains as an ansatz

$$
\begin{aligned}
\mathcal{O}\left(\alpha^{3}\right)-\mathcal{O}\left(\alpha_{\mathrm{t}}^{3}\right) & \sim \frac{\mathcal{O}\left(\alpha^{2}\right)-\mathcal{O}\left(\alpha_{\mathrm{t}}^{2}\right)}{\mathcal{O}(\alpha)} \mathcal{O}\left(\alpha^{2}\right), \\
\mathcal{O}\left(\alpha^{2} \alpha_{\mathrm{s}}\right)-\mathcal{O}\left(\alpha_{\mathrm{t}}^{2} \alpha_{\mathrm{s}}\right) & \sim \frac{\mathcal{O}\left(\alpha^{2}\right)-\mathcal{O}\left(\alpha_{\mathrm{t}}^{2}\right)}{\mathcal{O}(\alpha)} \mathcal{O}\left(\alpha \alpha_{\mathrm{s}}\right), \\
\mathcal{O}\left(\alpha \alpha_{\mathrm{s}}^{2}\right)-\mathcal{O}\left(\alpha_{\mathrm{t}} \alpha_{\mathrm{s}}^{2}\right) & \sim \frac{\mathcal{O}\left(\alpha \alpha_{\mathrm{s}}\right)-\mathcal{O}\left(\alpha_{\mathrm{t}} \alpha_{\mathrm{s}}\right)}{\mathcal{O}(\alpha)} \mathcal{O}\left(\alpha \alpha_{\mathrm{s}}\right), \\
\mathcal{O}\left(\alpha \alpha_{\mathrm{s}}^{3}\right)-\mathcal{O}\left(\alpha_{\mathrm{t}} \alpha_{\mathrm{s}}^{3}\right) & \sim \frac{\mathcal{O}\left(\alpha \alpha_{\mathrm{s}}\right)-\mathcal{O}\left(\alpha_{\mathrm{t}} \alpha_{\mathrm{s}}\right)}{\mathcal{O}(\alpha)} \mathcal{O}\left(\alpha \alpha_{\mathrm{s}}^{2}\right),
\end{aligned}
$$

where $\alpha_{\mathrm{t}}=y_{\mathrm{t}}^{2} /(4 \pi)$. Since we are only interested in the missing higher orders beyond the leading large- $y_{\mathrm{t}}$ limit, the same leading large- $y_{\mathrm{t}}$ approximations have been subtracted in the numerators on the right-hand sides.

The contribution of these estimates to the overall theory error evaluation is shown in table 8 for various pseudo-observables, and in table 9 for the $Z$-boson form factors. Note that the error estimate for $\sin ^{2} \theta_{\text {eff }}^{\ell}$ is slightly improved compared to refs. [28, 29] due to the inclusion of $\mathcal{O}\left(\alpha_{\mathrm{t}} \alpha_{\mathrm{s}}^{3}\right)$ corrections from refs. [79-81].

Nevertheless, we would also like to remind the reader that any estimate of the theory error from missing higher orders is not a precise prediction. Therefore it is generally desirable to ensure that the theory error is sub-dominant in any phenomenological analysis. Comparing the numbers in table 8 to current measurement results $[58,59]$, this is clearly seen to be the case. 


\begin{tabular}{|l|cccc|c|}
\hline Observable & $\alpha \alpha_{\mathrm{s}}^{2}$ & $\alpha \alpha_{\mathrm{s}}^{3}$ & $\alpha^{2} \alpha_{\mathrm{s}}$ & $\alpha^{3}$ & Total \\
\hline$\Gamma_{e, \mu, \tau}[\mathrm{MeV}]$ & 0.008 & 0.001 & 0.010 & 0.013 & 0.018 \\
$\Gamma_{\nu}[\mathrm{MeV}]$ & 0.008 & 0.001 & 0.008 & 0.011 & 0.016 \\
$\Gamma_{u, c}[\mathrm{MeV}]$ & 0.025 & 0.004 & 0.08 & 0.07 & 0.11 \\
$\Gamma_{d, s}[\mathrm{MeV}]$ & 0.016 & 0.003 & 0.06 & 0.05 & 0.08 \\
$\Gamma_{b}[\mathrm{MeV}]$ & 0.11 & 0.02 & 0.13 & 0.06 & 0.18 \\
$\Gamma_{\mathrm{Z}}[\mathrm{MeV}]$ & 0.23 & 0.035 & 0.21 & 0.20 & 0.4 \\
\hline$R_{\ell}\left[10^{-3}\right]$ & 2.5 & 0.4 & 3.6 & 3.9 & 6 \\
$R_{c}\left[10^{-5}\right]$ & 1.6 & 0.3 & 3.4 & 3.0 & 5 \\
$R_{b}\left[10^{-5}\right]$ & 5.5 & 0.9 & 6.4 & 3.7 & 10 \\
\hline$\sigma_{\text {had }}^{0}[\mathrm{pb}]$ & 0.2 & 0.03 & 4.2 & 3.7 & 6 \\
\hline $\sin ^{2} \theta_{\text {eff }}^{\ell}\left[10^{-5}\right]$ & - & 0.3 & 3.0 & 3.1 & 4.3 \\
$\sin ^{2} \theta_{\mathrm{eff}}^{b}\left[10^{-5}\right]$ & 0.7 & 0.4 & 4.3 & 3.2 & 5.3 \\
\hline
\end{tabular}

Table 8. Leading unknown higher-order corrections and their estimated order of magnitude for various pseudo-observables. The different orders always correspond to missing higher orders beyond the known approximations in the limit of a large top Yukawa coupling. The total theory error is obtained by adding the individual orders in quadrature.

\begin{tabular}{|l|cccc|c|}
\hline Observable & $\alpha \alpha_{\mathrm{s}}^{2}$ & $\alpha \alpha_{\mathrm{s}}^{3}$ & $\alpha^{2} \alpha_{\mathrm{s}}$ & $\alpha^{3}$ & Total \\
\hline$F_{V}^{\ell}\left[10^{-5}\right]$ & 0.03 & 0.004 & 0.2 & 0.4 & 0.5 \\
$F_{A}^{\ell}\left[10^{-5}\right]$ & 0.17 & 0.03 & 0.3 & 0.3 & 0.4 \\
$F_{V, A}^{\nu}\left[10^{-5}\right]$ & 0.16 & 0.02 & 0.3 & 0.5 & 0.6 \\
$F_{V}^{u, c}\left[10^{-5}\right]$ & 0.09 & 0.01 & 0.4 & 0.2 & 0.5 \\
$F_{A}^{u, c}\left[10^{-5}\right]$ & 0.17 & 0.03 & 0.3 & 0.4 & 0.5 \\
$F_{V}^{d, s}\left[10^{-5}\right]$ & 0.2 & 0.03 & 0.6 & 0.8 & 1.1 \\
$F_{A}^{d, s}\left[10^{-3}\right]$ & 0.3 & 0.04 & 0.4 & 0.5 & 0.7 \\
$F_{V}^{b}\left[10^{-5}\right]$ & 0.2 & 0.03 & 0.8 & 0.7 & 1.1 \\
$F_{A}^{b}\left[10^{-5}\right]$ & 0.3 & 0.04 & 0.1 & 0.1 & 0.3 \\
\hline
\end{tabular}

Table 9. Same as table 8, but for various form factors.

\section{Conclusions and outlook}

In this study, we have presented some phenomenologically useful applications of the recently completed electroweak two-loop calculation of $Z$-boson vertex corrections [36, 37]. The work collects multi-year efforts of several groups for predictions of the EWPOs related to the $Z$ peak up to electroweak full two-loop accuracy, supplemented by leading QCD higher-order terms. We have determined the two-loop electroweak contributions with a net relative numerical accuracy of about four digits. This ensures that these two-loop results 
will be known with sufficient accuracy even when adding the next perturbative order, as it might be needed for applications at the next generation of $e^{+} e^{-}$colliders.

For practical applications, the results for the EWPOs, as well as for the $Z$-boson vertex form factors, have been presented in terms of simple parameterization formulae, whose coefficients have been fitted to the full numerical computation. It is planned to include these fitting formulae into a new version of the weak library DIZET of ZFITTER $[45,46,48,88-90] .^{3}$ The accuracy of the fitting formulae is less than our full numerical two-loop calculation, but more than sufficient for present-day purposes. For the future FCC-ee Tera-Z project, it may be necessary to provide more precise formulae by including more terms with higher powers of the input parameters.

Finally, we would like to make a few comments on the prospects for the calculation of electroweak three-loop corrections, which will be necessary for the level of precision foreseen for FCC-ee and similar $e^{+} e^{-}$collider proposals. This electroweak third order, by itself, will be needed with only about two digits accuracy [91]. The generation of the amplitudes for $\mathcal{O}\left(10^{4}-10^{5}\right)$ diagrams as well as the evaluation of the Lorentz and Dirac algebra are routine tasks performed by computer algebra programs, and they should be straightforward with increased computing power in the future. Potential specific problems related to the treatment of $\gamma_{5}$ at three-loop level have to be controlled [92].

The most challenging problem will certainly be the stable numerical computation of three-loop Feynman integrals with several different internal mass scales. At two loops, we did not perform any reduction of the Feynman integrals to a smaller number of masters and thus had to calculate about 1000 previously unknown numerical integrals. In the next perturbative order, it may be advantageous to perform such a reduction to masters, given the ever increasing performance of programs like KIRA [93, 94], Reduze 2 [95], FIRE [96, 96], and LiteRED [97].

For the calculation of the (master) integrals themselves, it is desirable to have a procedure to automatically isolate and treat the ultra-violet and infra-red singularities. Although there is rapid progress in several analytical approaches to complicated loop integrals $[6,7]$, one has to expect that the more complicated ones will have to be done numerically. An additional complication is that for integrals with physical cuts, their stable numerical evaluation becomes more challenging. There are two kinds of software packages available that address these problems, based on either sector decomposition (SD) as realized in the SecDec project [98-103] and the FIESTA project [104-107], or based on Mellin-Barnes (MB) transformations, as implemented in the AMBRE project [108-121]. Sector decomposition is typically advantageous for integrals with many different mass scales, while the MB approach is more efficient for integrals with fewer independent parameters. Both methods certainly have room for crucial improvements. Several other numerical integration methods, as reviewed e.g. in refs. [6, 7], are useful for certain classes of multi-loop integrals, even though they are less general than the SD and MB approaches. Overall, numerical loop integration techniques are well positioned to meet the necessary future precision demands.

\footnotetext{
${ }^{3}$ Private communication by L. Kalinovskaya for the ZFITTER/DIZET support team.
} 
An electroweak three-loop result for the $Z$-peak EWPOs must be accompanied by improved calculations of the corrections needed to translate EWPOs to real observables. These include initial-state and final-state QED corrections and their interference as well as higher-order terms of the Laurent series expansion about the $Z$ resonance pole [6]. The latter will, for example, involve massive two-loop box diagrams. A complete accounting of the required correction terms is still lacking.

To summarize, we have completed the electroweak two-loop predictions for the EWPOs of the $Z$ resonance and collect here an extensive set of new fitting formulae for them. On a longer time scale, the calculation of the next perturbative order for the calculation of the EWPOs will be necessary and, with proper investments, realistically accessible.

\section{Acknowledgments}

The work of I.D. was supported by a research grant of Deutscher Akademischer Austauschdienst (DAAD) and by Deutsches Elektronensychrotron DESY and CERN. The work of J.G. was supported by the Polish National Science Centre (NCN) under the Grant Agreement 2017/25/B/ST2/01987 and by the program International mobilities for research activities of the University of Hradec Králové, CZ.02.2.69/0.0/0.0/16_027/0008487. The work of A.F. was supported in part by the National Science Foundation under grant no. PHY-1820760. The work of T.R. is funded by Deutsche Rentenversicherung Bund. He is supported in part by a 2015 Alexander von Humboldt Honorary Research Scholarship of the Foundation for Polish Sciences (FNP) and by the Polish National Science Centre (NCN) under the Grant Agreement 2017/25/B/ST2/01987. J.U. received funding from the European Research Council (ERC) under the European Union's Horizon 2020 research and innovation programme under grant agreement no. 647356 (CutLoops). The project was partly supported by the COST (European Cooperation in Science and Technology) Action CA16201 PARTICLEFACE. We finally acknowledge kind support for a working meeting by Prof. Peter Uwer at Humboldt-Universität zu Berlin.

Open Access. This article is distributed under the terms of the Creative Commons Attribution License (CC-BY 4.0), which permits any use, distribution and reproduction in any medium, provided the original author(s) and source are credited.

\section{References}

[1] A. Blondel, F. Dydak, A. Para and W. Blum, Measurement of the Z $Z^{0}$ Mass and Width at LEP, in Physics at LEP 1, Yellow Report CERN 86-02, Geneva Switzerland (1986).

[2] G. Arduini et al., LEP-1 operation, 1989-1995, Conf. Proc. C 960610 (1996) 286 [INSPIRE].

[3] J. Ellis and R. Peccei eds., Physics at LEP 1, Yellow Report CERN 86-02, Geneva Switzerland (1986), http://cds.cern.ch/record/166310/files/CERN-86-02-V-1.pdf.

[4] G. Altarelli, R. Kleiss and C. Verzegnassi eds., Z physics at LEP 1, Vol. 1: Standard physics, Vol. 2: Higgs search and new physics, Vol. 3: Event generators and software, Yellow Report CERN 89-08, Geneva Switzerland (1989), 
[5] D. Bardin, W. Hollik and G. Passarino eds., Reports of the working group on precision calculations for the $Z$ resonance, Yellow Report CERN 95-03, Geneva Switzerland (1995), parts I-III, http://cds.cern.ch/record/280836/files/CERN-95-03.pdf.

[6] A. Blondel et al. eds., Standard Model Theory for the FCC-ee: The Tera-Z, Report on the Mini Workshop on Precision EW and QCD Calculations for the FCC Studies: Methods and Techniques, CERN, Geneva Switzerland (2018) [arXiv: 1809.01830] [INSPIRE].

[7] A. Blondel, J. Gluza, S. Jadach, P. Janot and T. Riemann eds., Theory report on the $11^{\text {th }}$ FCC-ee workshop: Theory and Experiments, CERN, Geneva Switzerland (2019) [arXiv: 1905.05078].

[8] A. Freitas, J. Gluza, S. Jadach and T. Riemann, Loops, matrix elements, EWPOs, in Standard Model Theory for the FCC-ee: The Tera-Z, Report on the Mini Workshop on Precision EW and QCD Calculations for the FCC Studies: Methods and Techniques, CERN, Geneva Switzerland (2018), section C.2.

[9] B. Lynn and G.D. Starkman orgs., The Standard Model at 50 Years: A Celebratory Symposium, hosted by the Case Western Reserve University, Department of Physics, Cleveland U.S.A. (2018). Proceedings in preparation, https://indico.cern.ch/event/704471/timetable/.

[10] M. Consoli and A. Sirlin, The role of the one loop electroweak effects in $e^{+} e^{-} \rightarrow \mu^{+} \mu^{-}$, in Physics at LEP 1, Yellow Report CERN 86-02, Geneva Switzerland (1986).

[11] A. Sirlin, Theoretical considerations concerning the $Z^{0}$ mass, Phys. Rev. Lett. 67 (1991) 2127 [INSPIRE].

[12] S. Willenbrock and G. Valencia, On the definition of the $Z$ boson mass, Phys. Lett. B 259 (1991) 373 [INSPIRE].

[13] R.G. Stuart, Gauge invariance, analyticity and physical observables at the $Z^{0}$ resonance, Phys. Lett. B 262 (1991) 113 [INSPIRE].

[14] A. Sirlin, Observations concerning mass renormalization in the electroweak theory, Phys. Lett. B 267 (1991) 240 [INSPIRE].

[15] R.G. Stuart, General renormalization of the gauge invariant perturbation expansion near the $Z^{0}$ resonance, Phys. Lett. B 272 (1991) 353 [INSPIRE].

[16] R.G. Stuart, The Structure of the $Z^{0}$ resonance and the physical properties of the $Z^{0}$ boson, Phys. Rev. Lett. 70 (1993) 3193 [INSPIRE].

[17] H.G.J. Veltman, Mass and width of unstable gauge bosons, Z. Phys. C 62 (1994) 35 [INSPIRE].

[18] M. Passera and A. Sirlin, Radiative corrections to $W$ and quark propagators in the resonance region, Phys. Rev. D 58 (1998) 113010 [hep-ph/9804309] [INSPIRE].

[19] D. Yu. Bardin, P.K. Khristova and O.M. Fedorenko, On the Lowest Order Electroweak Corrections to Spin 1/2 Fermion Scattering. 1. The One Loop Diagrammar, Nucl. Phys. B 175 (1980) 435 [inSPIRE].

[20] D. Yu. Bardin, P.K. Khristova and O.M. Fedorenko, On the Lowest Order Electroweak Corrections to Spin 1/2 Fermion Scattering. 2. The One Loop Amplitudes, Nucl. Phys. B 197 (1982) 1 [inSPIRE].

[21] W.J. Marciano and A. Sirlin, Radiative Corrections to Neutrino Induced Neutral Current Phenomena in the $\mathrm{SU}(2)_{L} \times \mathrm{U}(1)$ Theory, Phys. Rev. D 22 (1980) 2695 [Erratum ibid. D 31 (1985) 213] [INSPIRE]. 
[22] W.J. Marciano and A. Sirlin, Testing the Standard Model by Precise Determinations of $W^{ \pm}$ and Z Masses, Phys. Rev. D 29 (1984) 945 [Erratum ibid. D 31 (1985) 213] [InSPIRE].

[23] A. Akhundov, D. Bardin and T. Riemann, Electroweak one-loop corrections to the decay of the neutral vector boson, Nucl. Phys. B 276 (1986) 1, preprint JINR-E2-85-617 (1985).

[24] J. Bernabeu, A. Pich and A. Santamaria, $\Gamma(Z \rightarrow b \bar{b}):$ A Signature of Hard Mass Terms for a Heavy Top, Phys. Lett. B 200 (1988) 569 [InSPIRE].

[25] F. Jegerlehner, Precision tests of electroweak interaction parameters, in: Proceedings of the 11th International School of Theoretical Physics: Testing the standard model, Szczyrk Poland (1987), R. Manka and M. Zralek eds., World Scientific, Singapore (1988), pg. 33, Bielefeld preprint BI-TP-87/16, https://lib-extopc.kek.jp/preprints/PDF/1988/8801/8801263.pdf

[26] W. Beenakker and W. Hollik, The Width of the Z Boson, Z. Phys. C 40 (1988) 141 [INSPIRE].

[27] M. Awramik, M. Czakon, A. Freitas and G. Weiglein, Complete two-loop electroweak fermionic corrections to $\sin ^{2} \theta_{\mathrm{eff}}^{\text {lept }}$ and indirect determination of the Higgs boson mass, Phys. Rev. Lett. 93 (2004) 201805 [hep-ph/0407317] [INSPIRE].

[28] M. Awramik, M. Czakon and A. Freitas, Bosonic corrections to the effective weak mixing angle at $O\left(\alpha^{2}\right)$, Phys. Lett. B 642 (2006) 563 [hep-ph/0605339] [INSPIRE].

[29] M. Awramik, M. Czakon and A. Freitas, Electroweak two-loop corrections to the effective weak mixing angle, JHEP 11 (2006) 048 [hep-ph/0608099] [INSPIRE].

[30] W. Hollik, U. Meier and S. Uccirati, The Effective electroweak mixing angle $\sin ^{2} \theta^{\mathrm{eff}}$ with two-loop fermionic contributions, Nucl. Phys. B 731 (2005) 213 [hep-ph/0507158] [INSPIRE].

[31] W. Hollik, U. Meier and S. Uccirati, The Effective electroweak mixing angle $\sin ^{2} \theta^{\mathrm{eff}}$ with two-loop bosonic contributions, Nucl. Phys. B 765 (2007) 154 [hep-ph/0610312] [INSPIRE].

[32] M. Awramik, M. Czakon, A. Freitas and B.A. Kniehl, Two-loop electroweak fermionic corrections to $\sin ^{2} \theta_{\mathrm{eff}}^{\mathrm{b} \overline{\mathrm{b}}}$, Nucl. Phys. B 813 (2009) 174 [arXiv:0811.1364] [InSPIRE].

[33] A. Freitas, Two-loop fermionic electroweak corrections to the Z-boson width and production rate, Phys. Lett. B 730 (2014) 50 [arXiv:1310.2256] [INSPIRE].

[34] A. Freitas, Electroweak precision tests in the LHC era and Z-decay form factors at two-loop level, in Proceedings of 12th DESY Workshop on Elementary Particle Physics: Loops and Legs in Quantum Field Theory (LL2014), Weimar Germany (2014) [arXiv: 1406.6980] [INSPIRE].

[35] A. Freitas, Higher-order electroweak corrections to the partial widths and branching ratios of the $Z$ boson, JHEP 04 (2014) 070 [arXiv:1401.2447] [INSPIRE].

[36] I. Dubovyk, A. Freitas, J. Gluza, T. Riemann and J. Usovitsch, The two-loop electroweak bosonic corrections to $\sin ^{2} \theta_{\mathrm{eff}}^{\mathrm{b}}$, Phys. Lett. B 762 (2016) 184 [arXiv:1607.08375] [INSPIRE].

[37] I. Dubovyk, A. Freitas, J. Gluza, T. Riemann and J. Usovitsch, Complete electroweak two-loop corrections to $Z$ boson production and decay, Phys. Lett. B $\mathbf{7 8 3}$ (2018) 86 [arXiv: 1804.10236] [INSPIRE].

[38] A. Leike, T. Riemann and J. Rose, $S$ matrix approach to the $Z$ line shape, Phys. Lett. B 273 (1991) 513 [hep-ph/9508390] [INSPIRE]. 
[39] T. Riemann, Cross-section asymmetries around the Z peak, Phys. Lett. B 293 (1992) 451 [hep-ph/9506382] [INSPIRE].

[40] S. Kirsch and T. Riemann, SMATASY: A program for the model independent description of the $Z$ resonance, Comput. Phys. Commun. 88 (1995) 89 [hep-ph/9408365] [InSPIRE].

[41] T. Riemann, S-matrix Approach to the Z Resonance, Acta Phys. Polon. B 46 (2015) 2235 [arXiv: 1610.04501] [INSPIRE].

[42] A. Freitas, J. Gluza and S. Jadach, QED deconvolution and pseudo-observables at FCC-ee precision, in Standard Model Theory for the FCC-ee: The Tera-Z, Report on the Mini Workshop on Precision EW and QCD Calculations for the FCC Studies: Methods and Techniques, CERN, Geneva Switzerland (2018), section C.3.

[43] S. Jadach and M. Skrzypek, QED challenges at FCC-ee precision measurements, arXiv: 1903.09895 [INSPIRE].

[44] AlePh, DelPhi, L3, OPAL, SLD, LeP Electroweak Working Group, SLD Electroweak Group and SLD Heavy Flavour Group collaborations, Precision electroweak measurements on the $Z$ resonance, Phys. Rept. 427 (2006) 257 [hep-ex/0509008] [INSPIRE].

[45] D. Yu. Bardin et al., ZFITTER v.6.21: A Semianalytical program for fermion pair production in $e^{+} e^{-}$annihilation, Comput. Phys. Commun. 133 (2001) 229 [hep-ph/9908433] [INSPIRE].

[46] A.B. Arbuzov et al., ZFITTER: A Semi-analytical program for fermion pair production in $e^{+} e^{-}$annihilation, from version 6.21 to version 6.42 , Comput. Phys. Commun. 174 (2006) 728 [hep-ph/0507146] [INSPIRE].

[47] M. Awramik, M. Czakon, A. Freitas and G. Weiglein, Precise prediction for the $W$ boson mass in the standard model, Phys. Rev. D 69 (2004) 053006 [hep-ph/0311148] [INSPIRE].

[48] A. Akhundov, A. Arbuzov, S. Riemann and T. Riemann, The ZFITTER project, Phys. Part. Nucl. 45 (2014) 529 [arXiv: 1302.1395] [INSPIRE].

[49] P.A. Baikov, K.G. Chetyrkin, J.H. Kühn and J. Rittinger, Complete $\mathcal{O}\left(\alpha_{s}^{4}\right) Q C D$ Corrections to Hadronic Z-Decays, Phys. Rev. Lett. 108 (2012) 222003 [arXiv:1201.5804] [INSPIRE].

[50] I. Dubovyk et al., Precision calculations for FCC, poster presented by S. Jadach at the FCC weak 2018, Amsterdam The Netherlands 2018, https://indico.cern.ch/event/656491/contributions/2947663/attachments/1622685/ 2582801/Poster-FCC-Amsterdam_SJadach_et_al.pdf.

[51] A. Abada et al., Future Circular Collider: Vol. 1: Physics opportunities, CERN-ACC-2018-0056 (2018).

[52] I. Dubovyk et al., Theory status of Z-boson physics, in Standard Model Theory for the FCC-ee: The Tera-Z, Report on the Mini Workshop on Precision EW and QCD Calculations for the FCC Studies: Methods and Techniques, CERN, Geneva Switzerland (2018), Chapter B.

[53] D. Yu. Bardin, A. Leike, T. Riemann and M. Sachwitz, Energy Dependent Width Effects in $e^{+} e^{-}$Annihilation Near the $Z$ Boson Pole, Phys. Lett. B 206 (1988) 539 [inSPIRE].

[54] D. Yu. Bardin, S. Riemann and T. Riemann, Electroweak One Loop Corrections to the Decay of the Charged Vector Boson, Z. Phys. C 32 (1986) 121 [INSPIRE]. 
[55] A. Denner and T. Sack, The W boson width, Z. Phys. C 46 (1990) 653 [InSPIRE].

[56] K.G. Chetyrkin, J.H. Kühn and A. Kwiatkowski, QCD corrections to the $e^{+} e^{-}$ cross-section and the $Z$ boson decay rate, in Reports of the working group on precision calculations for the $Z$ resonance, Yellow Report CERN 95-03, Geneva Switzerland (1995).

[57] K.G. Chetyrkin, J.H. Kühn and A. Kwiatkowski, QCD corrections to the $e^{+} e^{-}$ cross-section and the $Z$ boson decay rate: Concepts and results, Phys. Rept. 277 (1996) 189 [hep-ph/9503396] [INSPIRE].

[58] ALEPH, DELPHI, L3, OPAL and LEP EleCtroweak collaborations, Electroweak Measurements in Electron-Positron Collisions at W-Boson-Pair Energies at LEP, Phys. Rept. 532 (2013) 119 [arXiv:1302.3415] [INSPIRE].

[59] C. Patrignani et al., Review of Particle Physics, Chin. Phys. C 40 (2016) 100001.

[60] M. Steinhauser, Leptonic contribution to the effective electromagnetic coupling constant up to three loops, Phys. Lett. B 429 (1998) 158 [hep-ph/9803313] [INSPIRE].

[61] M. Davier, A. Hoecker, B. Malaescu and Z. Zhang, Reevaluation of the hadronic vacuum polarisation contributions to the Standard Model predictions of the muon $g-2$ and $\alpha\left(m_{Z}^{2}\right)$ using newest hadronic cross-section data, Eur. Phys. J. C 77 (2017) 827 [arXiv: 1706.09436] [INSPIRE].

[62] F. Jegerlehner, Variations on Photon Vacuum Polarization, arXiv:1711.06089 [INSPIRE].

[63] A. Keshavarzi, D. Nomura and T. Teubner, Muon $g-2$ and $\alpha\left(M_{Z}^{2}\right)$ : a new data-based analysis, Phys. Rev. D 97 (2018) 114025 [arXiv:1802.02995] [INSPIRE].

[64] A. Djouadi and C. Verzegnassi, Virtual Very Heavy Top Effects in LEP / SLC Precision Measurements, Phys. Lett. B 195 (1987) 265 [INSPIRE].

[65] A. Djouadi, $O\left(\alpha \alpha_{s}\right)$ Vacuum Polarization Functions of the Standard Model Gauge Bosons, Nuovo Cim. A 100 (1988) 357 [inSPIRE].

[66] B.A. Kniehl, Two Loop Corrections to the Vacuum Polarizations in Perturbative QCD, Nucl. Phys. B 347 (1990) 86 [InSPIRE].

[67] B.A. Kniehl and A. Sirlin, Dispersion relations for vacuum polarization functions in electroweak physics, Nucl. Phys. B 371 (1992) 141 [INSPIRE].

[68] A. Djouadi and P. Gambino, Electroweak gauge bosons selfenergies: Complete QCD corrections, Phys. Rev. D 49 (1994) 3499 [Erratum ibid. D 53 (1996) 4111] [hep-ph/9309298] [INSPIRE].

[69] A. Czarnecki and J.H. Kühn, Nonfactorizable QCD and electroweak corrections to the hadronic Z boson decay rate, Phys. Rev. Lett. 77 (1996) 3955 [hep-ph/9608366] [INSPIRE].

[70] R. Harlander, T. Seidensticker and M. Steinhauser, Complete corrections of Order $\alpha \alpha_{s}$ to the decay of the $Z$ boson into bottom quarks, Phys. Lett. B 426 (1998) 125 [hep-ph/9712228] [INSPIRE].

[71] J. Fleischer, O.V. Tarasov, F. Jegerlehner and P. Raczka, Two loop $O\left(\alpha_{s} G_{\mu} m_{t}^{2}\right)$ corrections to the partial decay width of the $Z_{0}$ into $b \bar{b}$ final states in the large top mass limit, Phys. Lett. B 293 (1992) 437 [INSPIRE].

[72] G. Buchalla and A.J. Buras, QCD corrections to the $\bar{s} d Z$ vertex for arbitrary top quark mass, Nucl. Phys. B 398 (1993) 285 [inSPIRE].

[73] G. Degrassi, Current algebra approach to heavy top effects in $Z \rightarrow b+\bar{b}$, Nucl. Phys. B 407 (1993) 271 [hep-ph/9302288] [INSPIRE]. 
[74] K.G. Chetyrkin, A. Kwiatkowski and M. Steinhauser, Leading top mass corrections of order $O\left(\alpha \alpha_{s} m_{T}^{2} / M_{W}^{2}\right)$ to partial decay rate $\Gamma(Z \rightarrow b \bar{b}) 4$, Mod. Phys. Lett. A 8 (1993) 2785 [INSPIRE].

[75] L. Avdeev, J. Fleischer, S. Mikhailov and O. Tarasov, $0\left(\alpha \alpha_{s}^{2}\right)$ correction to the electroweak $\rho$ parameter, Phys. Lett. B 336 (1994) 560 [Erratum ibid. B 349 (1995) 597] [hep-ph/9406363] [INSPIRE].

[76] K.G. Chetyrkin, J.H. Kühn and M. Steinhauser, Corrections of order $\mathcal{O}\left(G_{F} M_{t}^{2} \alpha_{s}^{2}\right)$ to the $\rho$ parameter, Phys. Lett. B 351 (1995) 331 [hep-ph/9502291] [INSPIRE].

[77] J.J. van der Bij, K.G. Chetyrkin, M. Faisst, G. Jikia and T. Seidensticker, Three loop leading top mass contributions to the $\rho$ parameter, Phys. Lett. B 498 (2001) 156 [hep-ph/0011373] [INSPIRE].

[78] M. Faisst, J.H. Kühn, T. Seidensticker and O. Veretin, Three loop top quark contributions to the $\rho$ parameter, Nucl. Phys. B 665 (2003) 649 [hep-ph/0302275] [INSPIRE].

[79] Y. Schröder and M. Steinhauser, Four-loop singlet contribution to the rho parameter, Phys. Lett. B 622 (2005) 124 [hep-ph/0504055] [INSPIRE].

[80] K.G. Chetyrkin, M. Faisst, J.H. Kühn, P. Maierhöfer and C. Sturm, Four-Loop QCD Corrections to the Rho Parameter, Phys. Rev. Lett. 97 (2006) 102003 [hep-ph/0605201] [INSPIRE].

[81] R. Boughezal and M. Czakon, Single scale tadpoles and $O\left(G_{F} m_{t}^{2} \alpha_{s}^{3}\right)$ corrections to the $\rho$ parameter, Nucl. Phys. B 755 (2006) 221 [hep-ph/0606232] [INSPIRE].

[82] P.A. Baikov, K.G. Chetyrkin and J.H. Kühn, Order $\alpha_{s}^{4} Q C D$ Corrections to $Z$ and tau Decays, Phys. Rev. Lett. 101 (2008) 012002 [arXiv:0801.1821] [INSPIRE].

[83] A.L. Kataev, Higher order $O\left(\alpha^{2}\right)$ and $O\left(\alpha \alpha_{s}\right)$ corrections to $\sigma_{\text {tot }}\left(e^{+} e^{-} \rightarrow\right.$ hadrons $)$ and Z-boson decay rate, Phys. Lett. B 287 (1992) 209 [INSPIRE].

[84] A. Abada et al., FCC Physics Opportunities, Eur. Phys. J. C 79 (2019) 474.

[85] CEPC Study Group collaboration, CEPC Conceptual Design Report: Volume 2 Physics \& Detector, arXiv:1811.10545 [INSPIRE].

[86] R. Hawkings and K. Monig, Electroweak and CP-violation physics at a linear collider $Z$ factory, Eur. Phys. J. direct 1 (1999) 8 [hep-ex/9910022] [INSPIRE].

[87] A. Freitas, Numerical multi-loop integrals and applications, Prog. Part. Nucl. Phys. 90 (2016) 201 [arXiv:1604.00406] [INSPIRE].

[88] A. Akhundov et al., Fortran program ZFITTER/DIZET v. 6.42, webpage http://sanc.jinr.ru/users/zfitter/.

[89] D. Yu. Bardin, M.S. Bilenky, G. Mitselmakher, T. Riemann and M. Sachwitz, A Realistic Approach to the Standard Z Peak, Z. Phys. C 44 (1989) 493 [inSPIRE].

[90] D. Yu. Bardin, M.S. Bilenky, T. Riemann, M. Sachwitz and H. Vogt, Dizet: A program package for the calculation of electroweak one loop corrections for the process $e^{+} e^{-} \rightarrow f^{+} f^{-}$ around the $Z^{0}$ peak, Comput. Phys. Commun. 59 (1990) 303 [inSPIRE].

[91] I. Dubovyk et al., Theory status of Z-boson physics, in Standard Model Theory for the FCC-ee: The Tera-Z, Report on the Mini Workshop on Precision EW and QCD Calculations for the FCC Studies: Methods and Techniques, CERN, Geneva Switzerland (2018), section B.1. 
[92] P. Marquard and D. Stöckinger, Towards 3- and 4-loop form factors, in Standard Model Theory for the FCC-ee: The Tera-Z, Report on the Mini Workshop on Precision EW and QCD Calculations for the FCC Studies: Methods and Techniques, CERN, Geneva Switzerland (2018), section D.1.

[93] P. Maierhöfer, J. Usovitsch and P. Uwer, Kira - A Feynman integral reduction program, Comput. Phys. Commun. 230 (2018) 99 [arXiv:1705.05610] [InSPIRE].

[94] P. Maierhöfer, J. Usovitsch, Recent developments in Kira, in Theory report on the $11^{\text {th }}$ FCC-ee workshop: Theory and Experiments, CERN, Geneva Switzerland (2019).

[95] A. von Manteuffel and C. Studerus, Reduze 2 - Distributed Feynman Integral Reduction, arXiv: 1201.4330 [INSPIRE].

[96] A.V. Smirnov, FIRE5: a C++ implementation of Feynman Integral REduction, Comput. Phys. Commun. 189 (2015) 182 [arXiv:1408.2372] [INSPIRE].

[97] R.N. Lee, LiteRed 1.4: a powerful tool for reduction of multiloop integrals, J. Phys. Conf. Ser. 523 (2014) 012059 [arXiv: 1310.1145] [INSPIRE].

[98] J. Carter and G. Heinrich, SecDec: A general program for sector decomposition, Comput. Phys. Commun. 182 (2011) 1566 [arXiv:1011.5493] [inSPIRE].

[99] S. Borowka, J. Carter and G. Heinrich, Numerical Evaluation of Multi-Loop Integrals for Arbitrary Kinematics with SecDec 2.0, Comput. Phys. Commun. 184 (2013) 396 [arXiv: 1204.4152] [INSPIRE].

[100] S. Borowka, G. Heinrich, S.P. Jones, M. Kerner, J. Schlenk and T. Zirke, SecDec-3.0: numerical evaluation of multi-scale integrals beyond one loop, Comput. Phys. Commun. 196 (2015) 470 [arXiv:1502.06595] [inSPIRE].

[101] S. Borowka et al., pySecDec: a toolbox for the numerical evaluation of multi-scale integrals, Comput. Phys. Commun. 222 (2018) 313 [arXiv:1703.09692] [INSPIRE].

[102] S. Borowka, G. Heinrich, S. Jahn, S.P. Jones, M. Kerner and J. Schlenk, A GPU compatible quasi-Monte Carlo integrator interfaced to pySecDec,

Comput. Phys. Commun. 240 (2019) 120 [arXiv:1811.11720] [InSPIRE].

[103] S. Jahn, SecDec: a toolbox for the numerical evaluation of multi-scale integrals, PoS (RADCOR2017) 017 [arXiv: 1802.07946] [INSPIRE].

[104] A.V. Smirnov and M.N. Tentyukov, Feynman Integral Evaluation by a Sector decomposiTion Approach (FIESTA), Comput. Phys. Commun. 180 (2009) 735 [arXiv: 0807.4129] [INSPIRE].

[105] A.V. Smirnov, V.A. Smirnov and M. Tentyukov, FIESTA 2: Parallelizeable multiloop numerical calculations, Comput. Phys. Commun. 182 (2011) 790 [arXiv:0912.0158] [INSPIRE].

[106] A.V. Smirnov, FIESTA 3: cluster-parallelizable multiloop numerical calculations in physical regions, Comput. Phys. Commun. 185 (2014) 2090 [arXiv:1312.3186] [INSPIRE].

[107] A.V. Smirnov, FIESTA4: Optimized Feynman integral calculations with GPU support, Comput. Phys. Commun. 204 (2016) 189 [arXiv:1511.03614] [INSPIRE].

[108] J. Gluza, K. Kajda and T. Riemann, AMBRE: A Mathematica package for the construction of Mellin-Barnes representations for Feynman integrals,

Comput. Phys. Commun. 177 (2007) 879 [arXiv:0704.2423] [InSPIRE]. 
[109] J. Gluza, K. Kajda, T. Riemann and V. Yundin, New results for loop integrals: AMBRE, CSectors, hexagon, PoS (ACAT08) 124 [arXiv: 0902.4830] [INSPIRE].

[110] J. Gluza, K. Kajda, T. Riemann and V. Yundin, Numerical Evaluation of Tensor Feynman Integrals in Euclidean Kinematics, Eur. Phys. J. C 71 (2011) 1516 [arXiv:1010.1667] [INSPIRE].

[111] J. Gluza, K. Kajda, T. Riemann and V. Yundin, News on Ambre and CSectors, Nucl. Phys. Proc. Suppl. 205-206 (2010) 147 [arXiv:1006.4728] [InSPIRE].

[112] J. Blümlein et al., Non-planar Feynman integrals, Mellin-Barnes representations, multiple sums, PoS (LL2014) 052 [arXiv: 1407.7832] [INSPIRE].

[113] I. Dubovyk, J. Gluza and T. Riemann, Non-planar Feynman diagrams and Mellin-Barnes representations with AMBRE 3.0, J. Phys. Conf. Ser. 608 (2015) 012070.

[114] I. Dubovyk, J. Gluza, T. Riemann and J. Usovitsch, Numerical integration of massive two-loop Mellin-Barnes integrals in Minkowskian regions, PoS(LL2016) 034 [arXiv: 1607.07538] [INSPIRE].

[115] I. Dubovyk, J. Gluza, T. Jelinski, T. Riemann and J. Usovitsch, New prospects for the numerical calculation of Mellin-Barnes integrals in Minkowskian kinematics, Acta Phys. Polon. B 48 (2017) 995 [arXiv: 1704.02288] [InSPIRE].

[116] I. Dubovyk, J. Gluza and T. Riemann, AMBRE - Construction of Mellin-Barnes integrals for two- and three-loop Z-boson decays, in Standard Model Theory for the FCC-ee: The Tera-Z, Report on the Mini Workshop on Precision EW and QCD Calculations for the FCC Studies: Methods and Techniques, CERN, Geneva Switzerland (2018), section E.4.

[117] I. Dubovyk, Mellin-Barnes representations for multiloop Feynman integrals with applications to 2-loop electroweak $Z$ boson studies, Ph.D. Thesis, Universität Hamburg, Hamburg Germany (2019).

[118] J. Usovitsch, I. Dubovyk and T. Riemann, MBnumerics: Numerical integration of Mellin-Barnes integrals in physical regions, PoS (LL2018) 046 [arXiv:1810.04580] [INSPIRE].

[119] J. Usovitsch et al., MBnumerics: Numerical integration of Mellin-Barnes integrals in physical regions, talk held by J. Usovitsch at LL2018, St. Goar Germany (2018). Slides: https://indico.desy.de/indico/event/16613/session/4/contribution/22/material/slides/0.pdf.

[120] J. Usovitsch, I. Dubovyk and T. Riemann, The MBnumerics project, in Standard Model Theory for the FCC-ee: The Tera-Z, Report on the Mini Workshop on Precision EW and QCD Calculations for the FCC Studies: Methods and Techniques, CERN, Geneva Switzerland (2018), section E.2.

[121] J. Usovitsch, Numerical evaluation of Mellin-Barnes integrals in Minkowskian regions and their application to two-loop bosonic electroweak contributions to the weak mixing angle of

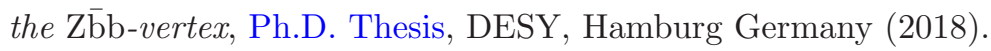

